Andrey I. Dmitriev, Lars B. Voll, Valentin L. Popov

\title{
The final NO-WEAR state due to dual- mode fretting: Numerical prediction and experimental validation
}

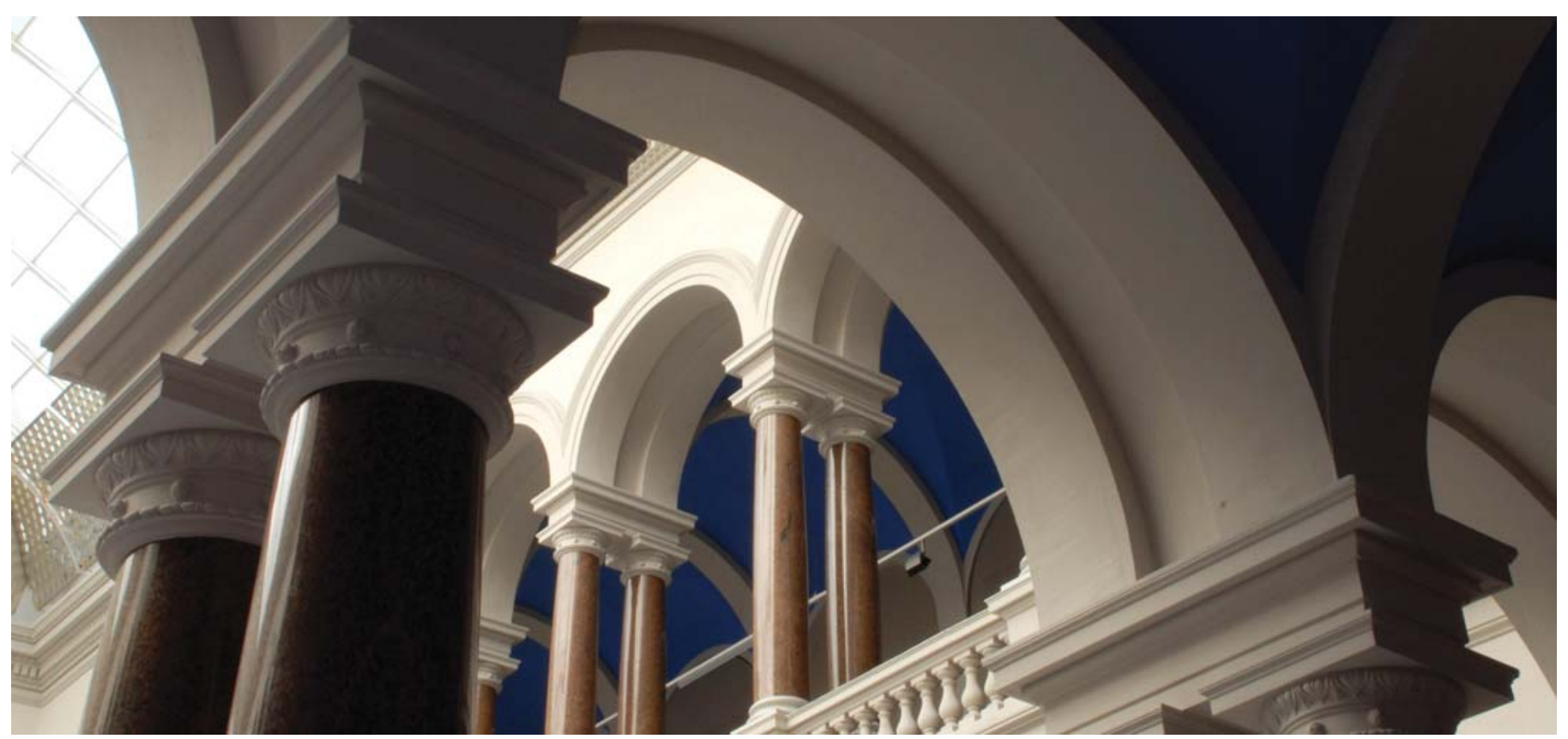

Dmitriev, A. I., Voll, L. B., \& Popov, V. L. (2020). The final NO-WEAR state due to dual-mode fretting: Numerical prediction and experimental validation. Wear, 458-459, 203402. https://doi.org/10.1016/j. wear.2020.203402 


\title{
THE FINAL NO-WEAR STATE DUE TO DUAL-MODE FRETTING: NUMERICAL PREDICTION AND EXPERIMENTAL VALIDATION
}

\author{
Andrey I. Dmitriev ${ }^{1,2, *}$, Lars B. Voll ${ }^{3}$, Valentin L. Popov ${ }^{1,2,3}$ \\ ${ }^{1}$ Institute of Strength Physics and Materials Science SB RAS, 634055, Tomsk, Russia \\ ${ }^{2}$ National Research Tomsk State University, 634050, Tomsk, Russia \\ ${ }^{3}$ Berlin University of Technology, 10623 Berlin, Germany \\ *Corresponding authors: $\underline{\text { dmitr@ispms.ru and v.popov@ @u-berlin.de }}$
}

\begin{abstract}
We study fretting wear due to superimposed oscillations in the normal and tangential directions with respect to the contact plane (dual mode fretting wear). In the limit of infinite time, the profile of the indenter tends to a limiting shape, which does not change further. For axisymmetric profiles, the limiting shape is found analytically for the general case of two different frequencies and amplitudes of oscillations in normal and tangential direction. The dependence of worn volume on the frequency ratio is strongly singular showing sharp minima for small rational ratios of frequencies. For the special case of coinciding frequencies, the fretting process was studied both analytically and experimentally. Comparison of experimental results with theoretical predictions showed a good qualitative and quantitative agreement (discrepancy of the order of experimental noise).
\end{abstract}

Keywords: fretting wear, method of dimensionality reduction, dual-mode fretting, experimental validation, no-wear state

\section{Introduction}

Fretting is wear caused by small amplitude oscillatory loading of static contact; alternating loads can be due to electromagnetic or mechanical vibration, or thermal cycling. Fretting was in the past an object of intensive experimental investigation and theoretical simulation for such applications as fretting of tubes in steam generators and heat exchangers $[1,2,3]$, joints in orthopedics [4], electrical connectors [5], and dovetail blade roots of gas turbines [6, 7] as well as many others. Most theoretical works were concerned with finite element $[8,9]$ or boundary element simulations [3]. A number of works have recently been devoted to numerical calculation of fretting wear using the so-called Method of Dimensionality Reduction (MDR) proposed in $[10,11]$.

According to the direction of relative motion of contacting bodies, four basic fretting modes can be distinguished: tangential, radial/normal, rotational and torsional fretting, as shown in Figure 1 .
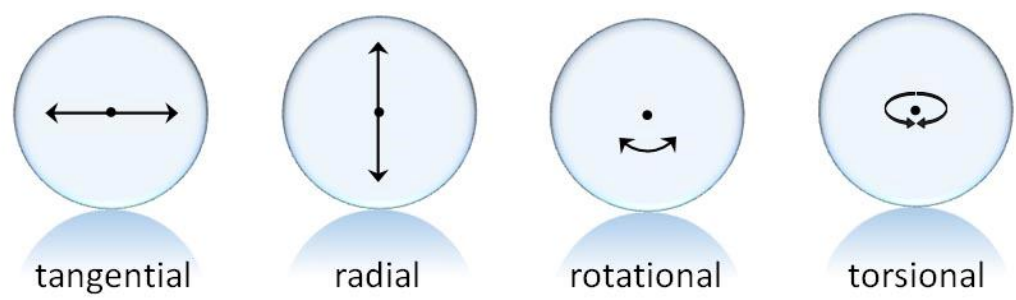

Figure 1. The scheme of four fretting modes

Up to now, the most studies of fretting were focused on the tangential mode $[12,13,14,15,16$, 17], there are very few reports about other fretting modes $[18,19,20]$ and even less about dualmotion fretting [21, 22]. The last one occurs by superposition of any two kinds of oscillation modes listed above [20] and includes a plenty of variations of phase portraits within the given degrees of freedom. In [20] the dual-motion fretting combining tangential and vertical oscillations of parabolic indenter was studied only at one of many possible variants of loading history, when both frequencies and amplitudes were equal and the phase shift was zero. At the 
same time the kind of wear and associated changes of indenter surface profile can vary depending on the certain parameters of oscillating functions. So, depending on particular properties of materials and on loading conditions, fretting can lead either to progressive wear or to some final state in which no further wear occurs. The existence of the limiting shape profile for three-dimensional contacts was theoretically proven in [23] and the final profiles for two examples of conical and parabolic indenters under conditions of tangential fretting were analytically calculated. Later it was experimentally validated in [24], where a special installation providing precise control of surface wear of the elastic hemisphere depending on the number of cycles under uniaxial tangential friction was developed to validate the analytical solution.

In the present paper, the developed setup was upgraded to enable experimental study of the fretting wear under oscillations in two perpendicular directions (normal and tangential) with an arbitrary ratio of the oscillation amplitudes. Thus, the main purpose of the present work is to prove analytically and confirm experimentally that for dual-mode fretting, combining arbitrary tangential and vertical small cyclic displacements of the indenter with axis-symmetric shape there are also conditions of the final, no-wear state. We would like to stress that the elastic coupling of the normal and tangential contacts may play an important role in fretting. In particular, according to Fig. 1, the "radial fretting" appears only in the presence of elastic coupling. The latter is governed by the second Dundurs' constant [10]. In particular, the Dundurs' constant is zero and the tangential and normal contacts are decoupled if one of the contact partners is rigid and the other is elastic but incompressible. These conditions are fulfilled in the experiments carried out in the present work.

\section{Theoretical background for the final state profile due to dual-mode fretting wear}

The calculation of the final state profile of the indenter surface as a result of dual-mode fretting wear can be carried out using the method of dimensionality reduction [10]. The governing equations of the method allow mapping of the 3D problem for an axisymmetric body, described in cylindrical coordinate system by $z=f(r)$, to a one-dimensional one. For a parabolic indenter, the initial 3D profile and resulting 1D function $g(x)$ used in the MDR are shown in Figure 2.

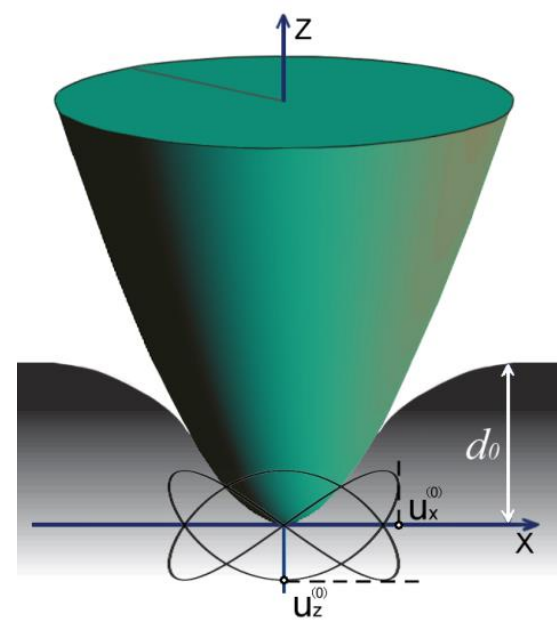

(a)

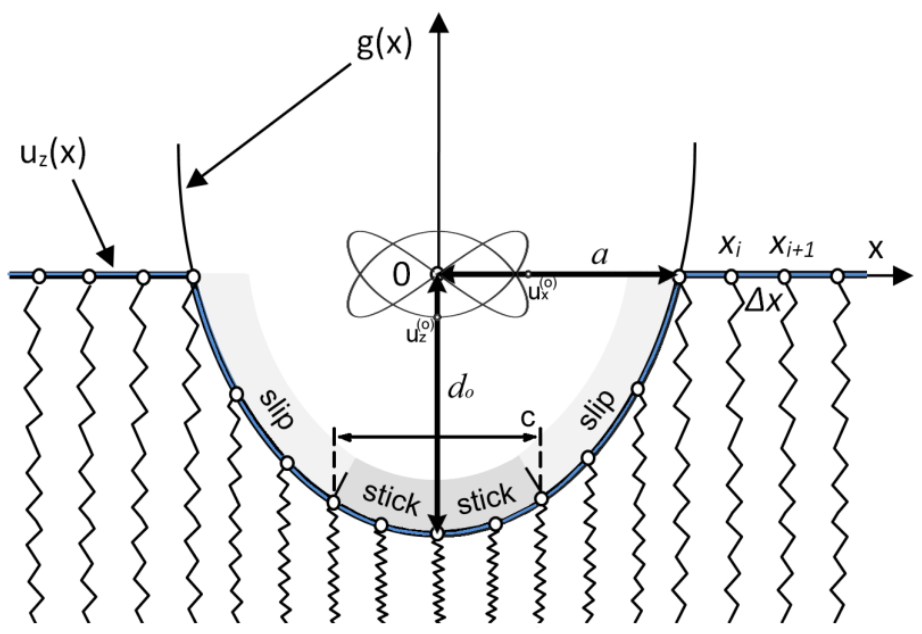

(б)

Figure 2. (a) A three-dimensional profile $f(r)$ and $(b)$ its $1 D$ representation $g(x)$.

If the indenter is moved tangentially and vertically by oscillating functions $u_{x}=u_{x}^{(0)} \sin \omega_{x} \Delta t$ and $u_{z}=u_{z}^{(0)} \sin \left(\omega_{z} \Delta t+\psi\right)$, the springs (see Fig. 2b) will be stressed both in the tangential and normal directions. The radius $c$ of the stick region will be given by the condition that the tangential force is less than the coefficient of friction $\mu$ multiplied by the normal force for all variable parameters $\omega_{x}, \omega_{z}, u_{x}^{(0)}, u_{z}^{(0)}$ and $\psi$ : 


$$
G^{*} u_{x}^{(0)} \sin \left(\omega_{x} t\right)<\mu E^{*}\left(d_{0}+u_{z}^{(0)} \sin \left(\omega_{z} t+\psi\right)-g(x)\right) .
$$

Otherwise it corresponds to slip conditions. Here $d_{0}$ is the controlled depth, $E^{*}$ and $G^{*}$ are the effective elastic and shear moduli. In order to estimate $g(x)$ we can rewrite (1) as:

$$
g(x)<d_{0}+u_{z}^{(0)} \sin \left(\omega_{z} t+\psi\right)-\frac{G^{*}}{\mu E^{*}} u_{x}^{(0)} \sin \left(\omega_{x} t\right)
$$

As was shown in [23], the pressure outside the stick area must vanish in the limiting shakedown state: $p(r)=0$, for $r>c$. This means that

$$
g^{\prime}(x)=0, \text { and } g^{\prime}(x)=g_{0}=\text { const }, \text { for } c<x<a,
$$

where $a$ is the radius of contact area.

Setting the derivative of the right-hand side of the inequality (2) equal zero results in the following equation:

$$
\cos \left(\omega_{z} t^{*}+\psi\right)=\frac{G^{*}}{\mu E^{*}} \frac{u_{x}^{(0)} \omega_{x}}{u_{z}^{(0)} \omega_{z}} \cos \left(\omega_{x} t^{*}\right),
$$

where a set of time moments $t^{*}$ is used to find the radius $c$ of the stick region for all variable parameters $\omega_{x}, \omega_{z}, u_{x}^{(0)}, u_{z}^{(0)}$ and $\psi$ from the following conditions:

$$
g(c)=\min \left\{d_{0}+u_{z}^{(0)} \sin \left(\omega_{z} t^{*}+\psi\right)-\frac{G^{*}}{\mu E^{*}} u_{x}^{(0)} \sin \left(\omega_{x} t^{*}\right)\right\}
$$

If the phase shift is not fixed, then the solution of Eq. (5) will be written as

$$
g(c)_{\min }=d_{0}-u_{z}^{(0)}-\frac{G^{*}}{\mu E^{*}} u_{x}^{(0)}
$$

Otherwise $g(c)_{\min }$ depends on the frequencies ratio $\omega_{z} / \omega_{x}$ and can be found numerically.

Let us denote the initial, non-worn shape of the indenter as $f_{0}(r)$, the corresponding onedimensional image as $g_{0}(x)$ and the limiting shakedown shapes as $f_{\infty}(r)$ and $g_{\infty}(x)$ correspondingly. So, the one-dimensional profile $g_{\infty}(x)$ in the limiting shape due to dual-motion fretting has the form

$$
g_{\infty}(x)= \begin{cases}g_{0}(x), & \text { for } 0<x<c \\ d_{\max }, & \text { for } c<x<a\end{cases}
$$

where $d_{\max }=d_{0}+u_{z}^{(0)}$. The resulting shape is schematically shown in Figure 3.

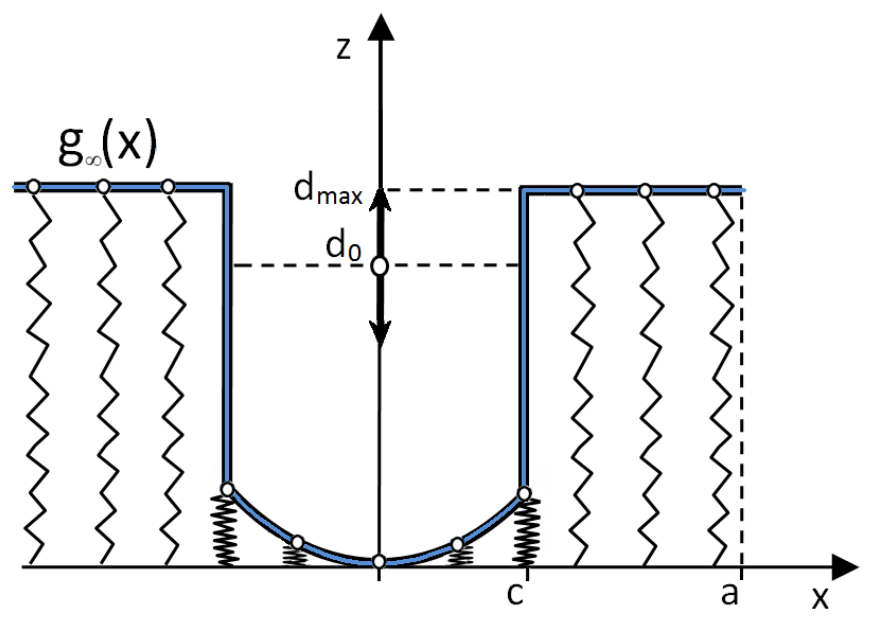

Figure 3. One dimensional limiting shakedown profile for dual-motion oscillations 
The corresponding three-dimensional limiting shape $f_{\infty}(r)$ can now be calculated by the back Abel transformation [25]:

$$
f_{\infty}(r)= \begin{cases}f_{0}(r), & \text { for } 0<r<c \\ \frac{2}{\pi} \int_{0}^{c} \frac{g_{0}(x)}{\sqrt{r^{2}-x^{2}}} d x+\frac{2}{\pi} d \int_{c}^{r} \frac{1}{\sqrt{r^{2}-x^{2}}} d x, & \text { for } c<r\end{cases}
$$

where $g_{0}(x)$ is given by the equation

$$
g_{0}(x)=|x| \int_{0}^{|x|} \frac{f_{0}^{\prime}(r)}{\sqrt{x^{2}-r^{2}}} \mathrm{~d} r
$$

and $f_{0}^{\prime}(r)$ means derivation by $r$. External radius $a$ of the worn area is given by the intersection of the profile (8) with the initial one. Note that the limiting shape depends on the initial shape, the radius of the stick area and the maximum indentation depth, but not on the elastic properties of the contacting bodies.

\section{Calculation of resulting wear volume}

Let us consider the parabolic indenter described by $f_{0}(r)=r^{2} /(2 R)$. In this case the radius $c$ of the stick area will be found as $c=\sqrt{\operatorname{Rg}(c)_{\min }}$. Figure 4 shows the final profiles (fig. 4a) and the resulting wear volume (fig. $4 \mathrm{~b}$ ) calculated during the tangential and normal oscillations of the indenter until achieving the no-wear state if the frequency ratio $\omega_{z} / \omega_{x}$ varies in the range $0.02 \div 1.0$ with an increment of 0.02 and both amplitudes $u_{z}^{(0)}$ and $u_{x}^{(0)}$ are equal. According to the results, there are three local well pronounced minima belonging to frequency relationships of $0.2,0.5$ and 1.0, where in the case of $\omega_{z}=\omega_{x}$ the wear is almost zero. On the other hand, almost all final profiles at other ratio values are very close to each other and form a wide black curve depicted in Figure 4a. Most calculated points of the reduced wear volume are located between $17 \times 10^{3}$ and $16 \times 10^{3}$. Only couple of them (e.g. corresponding to frequency relationships of 0.4 and 0.8 ) drop below $15 \times 10^{3}$. Note that in our calculations the wear volume $\tilde{\Omega}=1$ corresponds to a basic amount of wear volume which is produced until achieving the limiting shape profile during the pure tangential oscillations.

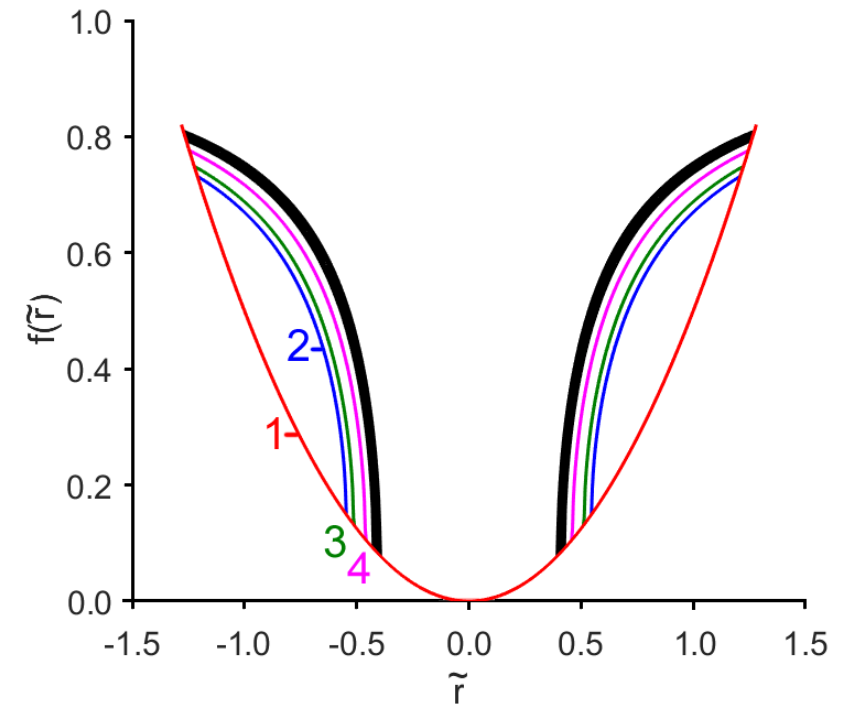

(a)

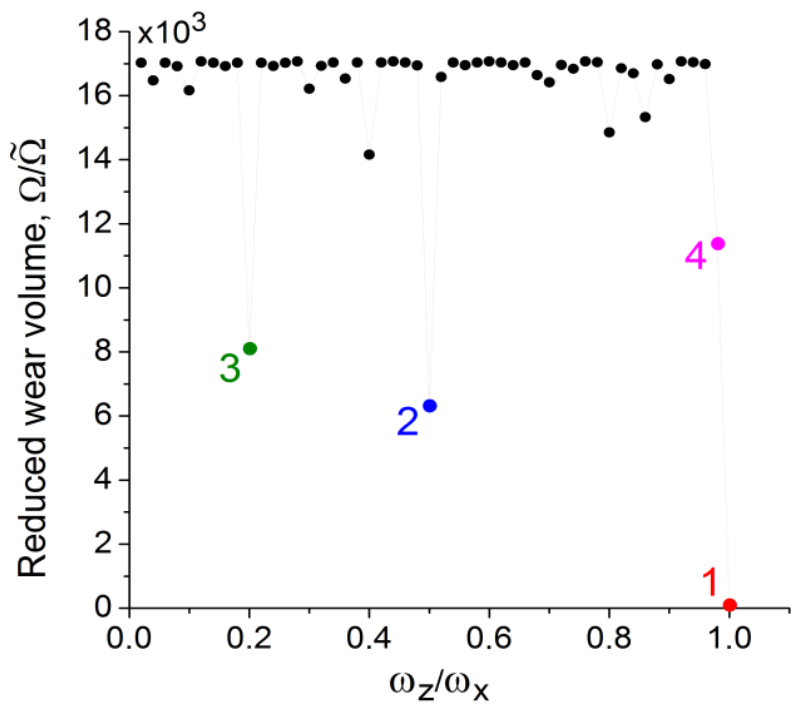

(b)

Figure 4. (a) The cross section of indenter final worn profiles, and (b) the resulting reduced wear volume for dual-motion fretting until shakedown state for frequencies dependence while 
$u_{z}^{(0)}=u_{x}^{(0)}$. Colors of curves in figure (a) correspond to the points of the same color in fig. (b).

Hereafter the wear volume $\tilde{\Omega}$ in case of $u_{z}^{(0)}=0$ (pure tangential oscillations) is used as a basic amount. Numbers in figures $(a)$ and $(b)$ correspond to the simple rational ratios $0.2,0.5$ and 1.0 as well one further point very close to " 1 " to illustrate the high sensitivity of the worn volume to the frequency ratio.

Let us discuss in a more detail the physical nature of the "strange" oscillations of the total wear which look in Figure 4(b) like a "noise". According to the present theory, the worn volume is unambiguously defined by the radius $c$ of the permanent stick, the latter being defined by the minimum indentation depth which is achieved during the oscillation process and is given by Eq. (5). If the ratio of frequencies $\omega_{x}$ and $\omega_{z}$ is irrational, then the quantity

$$
d_{0}+u_{z}^{(0)} \sin \left(\omega_{z} t^{*}+\psi\right)-\frac{G^{*}}{\mu E^{*}} u_{x}^{(0)} \sin \left(\omega_{x} t^{*}\right)
$$

will accept during long enough time any values between

$$
d_{0}-\left|u_{z}^{(0)}\right|-\frac{G^{*}}{\mu E^{*}}\left|u_{x}^{(0)}\right| \quad \text { and } \quad d_{0}+\left|u_{z}^{(0)}\right|+\frac{G^{*}}{\mu E^{*}}\left|u_{x}^{(0)}\right|,
$$

independently on the phase shift $\psi$. The minimum value of $c$ is then given by Equation (6) and represents the smallest indentation depth possible for the given oscillation amplitudes corresponding to the maximum possible wear volume. For any rational ratio of $\omega_{x}$ and $\omega_{z}$, this will not be the case as not all points of the above interval can be reached. For rational frequency ratio the process will be periodic. However, any, even "infinitesimal small" change of one of frequencies will lead to a final change of the period and the space of positions of indenter which can be reached during the oscillation. Thus, the wear volume as function of the frequencies ratio is, strictly speaking, a function which is not continuous in any point (has the "Weierstrassstructure"). Of course, these singularity in any point has no practical importance as the differentiation between rational and irrational values cannot be realized physically due to always present small variations of conditions. Interestingly, very similar situation does exist for the states of electrons in a periodic crystal lattice in the presence of magnetic field, where the energetic sub-bands appearing in magnetic field are extremely sensitive to the magnitude and direction of the magnetic field [26]. In the quantum theory of electrons in crystal lattices this exact electronic structure is just "ignored". Landau and Lifshitz explain this as follows "It must be emphasized, however, that this does not imply a similar instability in observable physical properties. These are determined not by specific band structure, but by the distribution of the number of states among small but finite energy ranges, and this distribution changes only slightly with the field...". This argument is very similar to our argument of always present small fluctuations.

\section{Description of the experimental set-up}

For experimental validation of the analytically predicted universal limiting shape profiles, an experimental set-up designed and described in [24] was modified according to the scheme depicted in Figure 5a. A general view of the set-up is given in Figure 5b. The development of the set-up was focused on ability to provide a freely selectable direction of the oscillation and a defined sample displacement. The amplitude of the oscillation was fixed at $0.8 \mathrm{~mm}$. For this purpose, a penetration depth $(1 \mathrm{~mm})$ was determined from preliminary experiments, so that the contact region is subdivided into stick and sliding regions. For simplicity, a hemisphere of elastomer was used as a sample. In this case, the dimensions of the sticking and sliding regions are macroscopic. Abrasive paper was chosen as a contact partner, so that the wear occurred rapidly and intensively. 


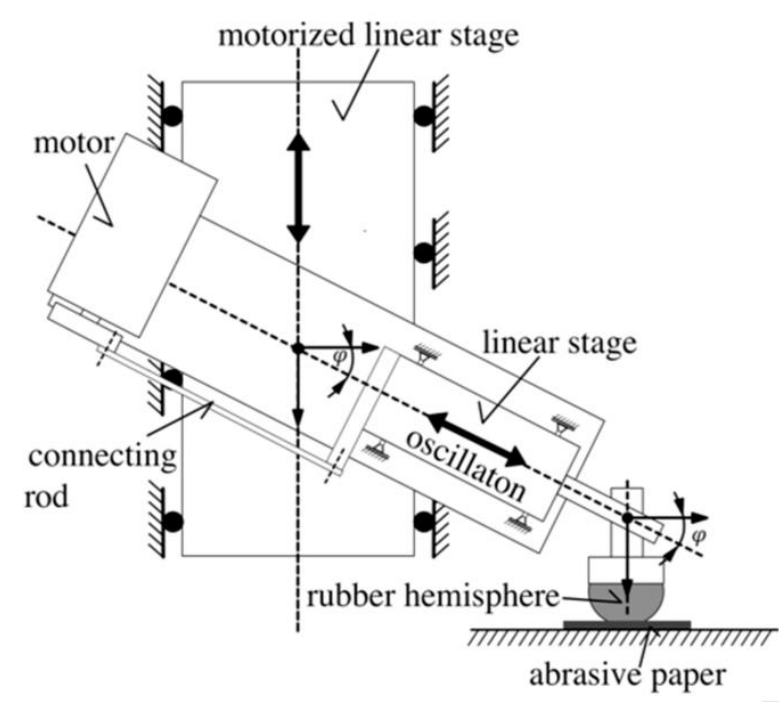

(a)

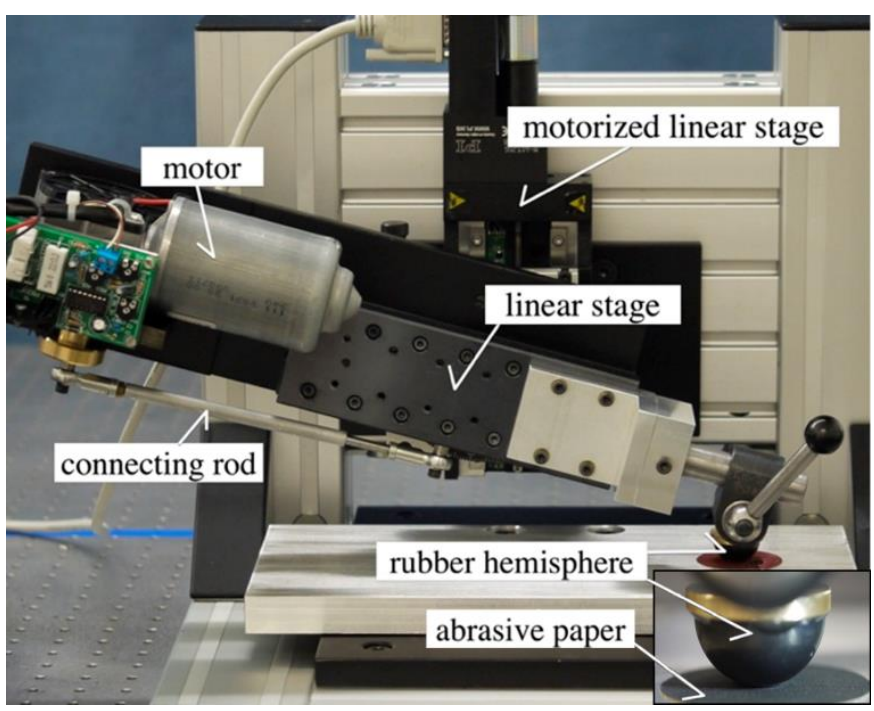

(b)

Figure 5. A principle sketch (a) and a general view (b) of the central part of the experimental set-up for studying dual-motion fretting wear. The rotational movement of the motor is converted to an oscillation of the linear stage using a connecting rod and an eccentric disc. The amplitude of the oscillations was $0.8 \mathrm{~mm}$ for all experiments with different angles. An enlarged image of a rubber half-ball indenter is given in the lower right corner of figure (b).

In order to reproduce the dual-mode fretting wear, the experimental set-up provided the possibility of changing the angle of inclination of the holder. So, the tangent of the given angle corresponded to the value of the amplitude ratio $u_{z}^{(0)} / u_{x}^{(0)}$, while both frequencies $\omega_{z}$ and $\omega_{x}$ were equal. With chosen angle the rubber half-ball was indented by a given indentation depth. After that the indenter was moved forward and back with fixed amplitude. After 2160 cycles, the sample was demounted and the shape was determined from photographs. Then the ball was mounted back, pressed to the same indentation depth, and loaded by tangential oscillations with the same for the next 2160 cycles and so on. The resulting shape of the indenter with progressive wear for the tangential fretting mode is shown in Figure 6. The parameters of the experiment were: ball radius $(R) 14.5 \mathrm{~mm}$, indentation depth $(d) 1 \mathrm{~mm}$, radius of the region of permanent stick (c) $2.45 \mathrm{~mm}$. The resulting outer radius $(a)$ of the worn region was $5.5 \mathrm{~mm}$.

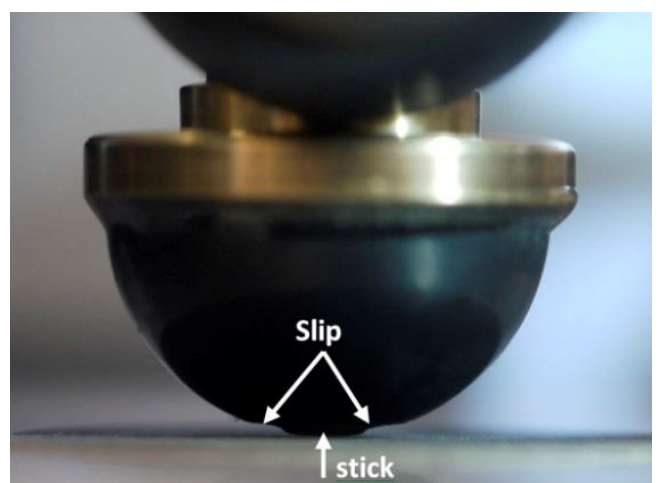

(a)

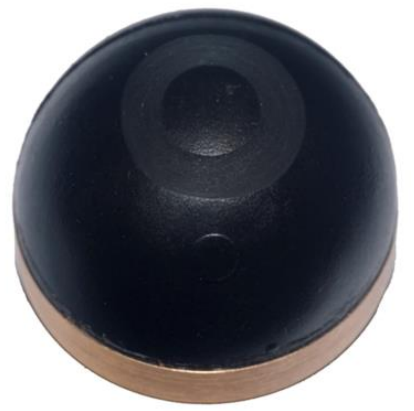

(b)

Figure 6. A side (a) and a top (b) view of indenter after tangential fretting-wear in a final no more wear state. Stick and slips areas are shown by arrows.

\section{Experimental determination of the limiting shape.}

The aim of the experimental investigation was to determine the wear profile that occurs after $N$ oscillation cycles as a function of the angle $\varphi$. The wear profile was measured at $\varphi=0^{\circ}, 7.5^{\circ}$, $15^{\circ}, 22.5^{\circ}, 30^{\circ}$ and $45^{\circ}$ with a laser confocal microscope. The experimental procedure was 
determined on the basis of a preliminary test series. After the adjustment of the angle $\varphi$ and the alignment of the sample, the elastomer hemisphere was pressed into the rigid substrate. After a holding time of 1 hour the sample was relieved. This procedure minimized viscoplastic deformation that occurred during the experiment. Then the motorized linear stage was moved vertically until the sample had the first contact with the abrasive paper. Starting from this position, the sample was pressed $1 \mathrm{~mm}$ into the abrasive paper. Then the oscillation was started at the angle $\varphi$. For cleaning or changing the abrasive paper the experiment was stopped. After each change the starting position of the sample was checked and readjusted if necessary. The procedure was repeated until the final profile was reached (no macroscopic wear). This process was repeated for all angles. The images of the laser scanning microscopy with the 3D final shape of indenter for considered angles are shown in Figure 7.
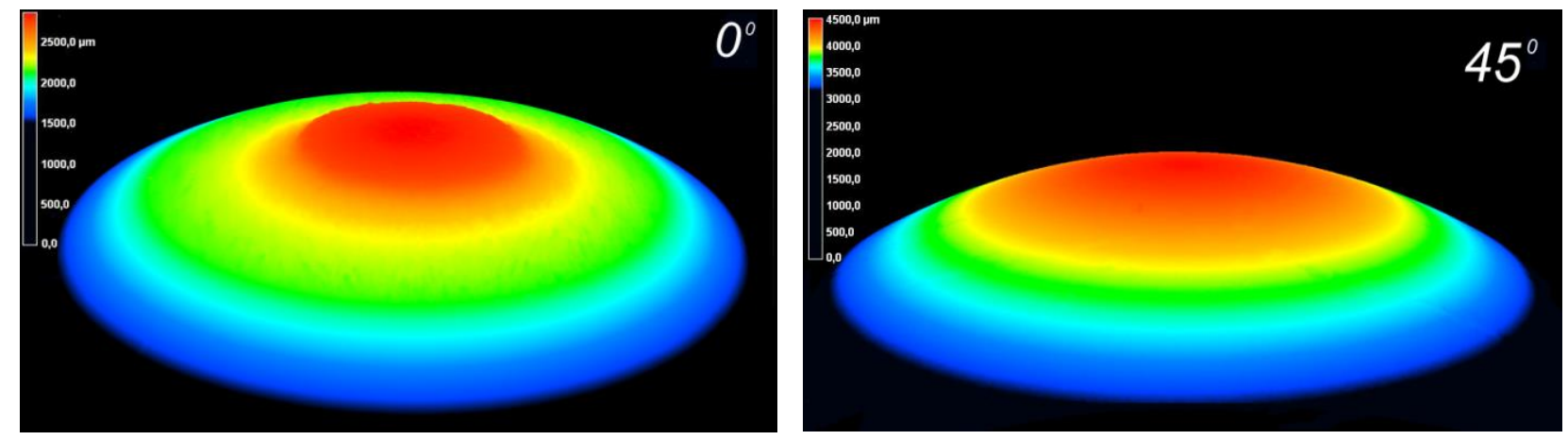

Figure 7. Two examples of 3D surfaces of indenters due to fretting caused by linear oscillations with inclination at angles of 0 and 45 degrees. See supplementary material using the following link: https://youtu.be/T66HVVKCK90.

\section{Data comparison}

According to spherical shape of the indenter we used for the calculation of the limiting shape shown in Figure 8 the exact ball form:

$$
f_{0}(r)=R-\sqrt{R^{2}-r^{2}}
$$

The limiting shape is determined by Eq. (8), where the function $g_{0}(x)$ can be calculated by substituting (10) into (9):

$$
g_{0}(x)=|x| \int_{0}^{|x|} \frac{r d r}{\sqrt{R^{2}-r^{2}} \sqrt{x^{2}-r^{2}}}=|x| \operatorname{artanh}\left(\frac{|x|}{R}\right)
$$

Thus, the explicit form of the limiting shape for the case, when the initial shape was a ball with radius $R$, is given by

$$
f_{\infty}(r)= \begin{cases}f_{0}(r), & \text { for } 0<r<c \\ \frac{2}{\pi} \int_{0}^{c} \frac{x \cdot \operatorname{artanh}(x / R)}{\sqrt{r^{2}-x^{2}}} \mathrm{~d} x+d_{\max }\left(1-\frac{2}{\pi} \arcsin \frac{c}{r}\right), & \text { for } c<r<a\end{cases}
$$

Relation (12) calculated for the case of pure tangential oscillation is presented in Figure 8a with a gray line. The measured shape after 8640 oscillation cycles (shown by yellow curve) amazingly well agrees with the predicted limiting shape. Other experimental and calculation data are summarized in comparison in Figure $8 \mathrm{~b}$. One can see pretty good agreement of experimental dots corresponding to reduced wear volume calculated on the basis of color lines shown in Figure $8 \mathrm{a}$ with the analytical solution. According to this dependence, the wear volume nonlinearly goes down with increasing a slope angle of the holder until it reaches the wear-free behavior at an angle $\varphi$ of $45^{\circ}$. 


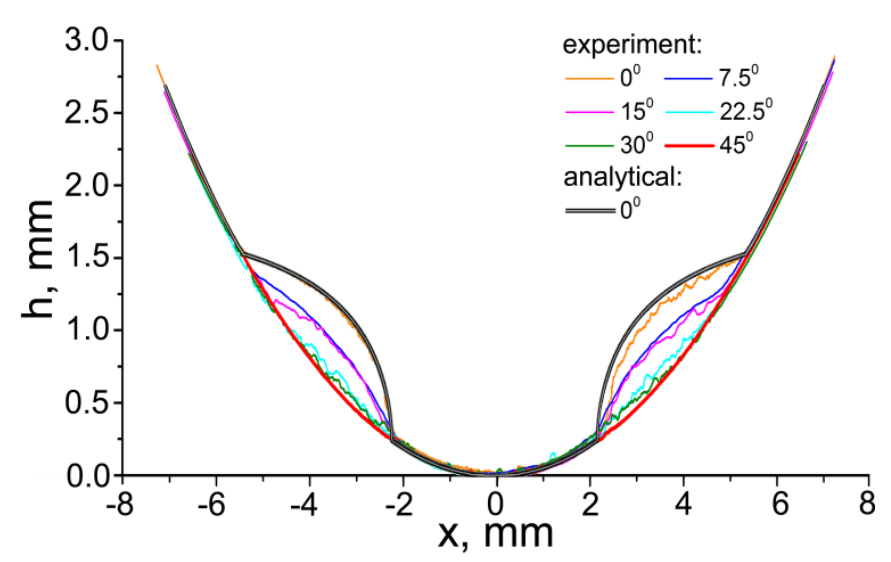

(a)

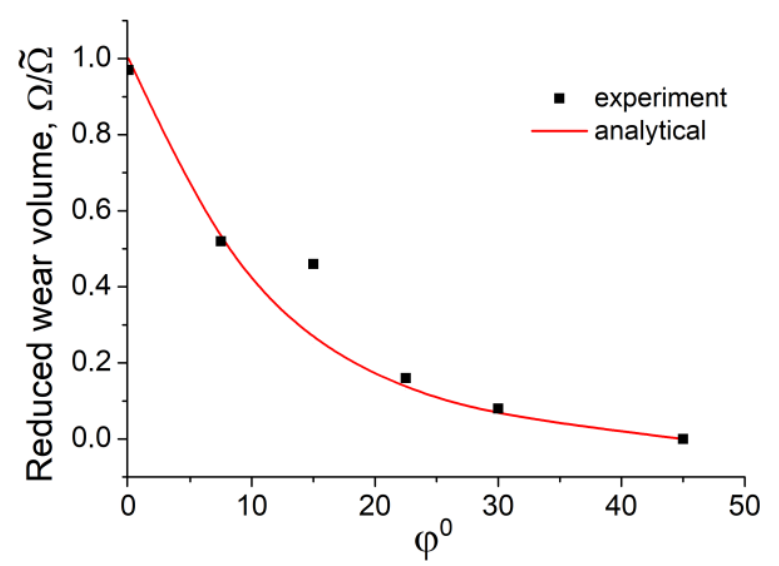

(b)

Figure 8. (a) Cross sections of spherical indenter final worn profiles experimentally obtained under tangential fretting wear at six slope angles of the holder. The theoretical limiting worn profile calculated according to Eq. (12) for the same parameters as in the experiment is shown by a grey curve. (b) Dependence of a relative wear volume obtained from the experimental data on the slope angle of the holder and the analytical values calculated according to Eq. (12) vs. amplitude ratio recalculated according to $\varphi=\arctan \left(u_{z}^{(0)} / u_{x}^{(0)}\right)$.

\section{Conclusions}

As previously shown in [23], the limiting shape does not depend on the type of fretting loading and on elastic properties of contacting bodies but is solely related to the initial shape in the region of permanent stick $f_{0}(r)$, the radius of the region of permanent stick, $c$, and the maximum indentation depth during the oscillation history, $d_{\max }$. In the present paper this statement was verified by an example of various implementations of dual-mode fretting wear. If we know the axisymmetric function that describes the indenter profile and both parameters that determine the history of the indenter motion in two perpendicular directions, then the final profile of such a body is unambiguously predicted in the framework of the MDR theory [10]. In particular cases, this solution is found analytically, or in more general examples it is calculated numerically, when there is no simple analytical solution. The universality was illustrated by a numerical example of complicated kinetics of the worn profile due to normal and tangential oscillations with different frequencies and amplitude relationships and was validated by the specially designed experimental test with tangential fretting under different slope angles of applied load. The presented results showed an excellent agreement between the experimental results and theoretical prediction.

Note that in the course of theoretical predictions of the volume of the worn surface, a nontrivial solution was found that shows the nature of the change in the worn profile depending on the frequencies ratio in the normal and tangential directions. Moreover, the equality of the oscillating frequencies leads to a significant (by several orders of magnitude) decrease in the fretting wear volume. The latter should be taken into account when designing various friction units experiencing oscillating nature of the effects. From the point of view of wearless contact pairs, an interesting case is, when, in addition to the equality of frequencies, there is also the equality of oscillation amplitudes. Such a limiting case, as predicted theoretically and demonstrated experimentally, is characterized by complete absence of wear associated with fretting. This is due to the fact that the contact area is constant in the conditions of a permanent stick. Thus, tangential fretting contact can be made as wear-free as possible by changing the relative angle of inclination of the contacting pair toward $45^{\circ}$. In this case, due to the nonlinear dependence, a decrease in the angle of inclination of the contacting pair within only $10^{\circ}$ makes it possible to reduce the volume of the worn surface by at least twice. 
Note also that the limiting profile described in the paper develops under very general assumptions. However even these general assumptions can still be violated under real experimental or technological conditions. One of these assumptions is the "assumption of continuous wear". This means that no wear particles are produced during the fretting process which size is comparable with the characteristic indentation depth. We have made sure that our experimental conditions meet these requirements. Another restriction of the present consideration is the absence of progressive plastic deformation in the final worn state. However, it is known that in metallic contacts these conditions can be violated due to adhesive wear. The suggested theory is not applicable to such cases. However, even in these cases, the existence of the limiting shape can be used for "soft preprocessing" under conditions in which the severe wear is avoided.

\section{Acknowledgements:}

This work was partially supported by Deutsche Forschungsgemeinschaft (DFG, PO 810-53-1), Deutscher Akademischer Austausch Dienst (DAAD), the Russian Fundamental Research Program of the State Academies of Sciences for 2013-2020 (Priority direction III.23.2.4) and the Tomsk State University (TSU) competitiveness improvement program.

\section{References:}

1. P.L. Ko, Experimental Studies of Tube Frettings in Steam Generators and Heat Exchangers, Journal of Pressure Vessel Technology 101 (1979) 125-133.

2. N.J. Fisher, A.B. Chow, M.K. Weckwerth, Experimental Fretting Wear Studies of Steam Generator Materials, Journal of Pressure Vessel Technology 117 (1995) 312-320.

3. C.Y. Lee, L.S. Tian, J.W. Bae, Y.S. Chai, Application of influence function method on the fretting wear of tube-to-plate contact, Tribology International 42 (2009) 951-957.

4. J.P. Collier, M.B. Mayor, R.E. Jensen et al., Mechanisms of failure of modular prostheses. Clinical Orthopaedics and Related Research 285 (1992) 129-139.

5. M. Antler, Electrical effects of fretting connector contact materials: a review, Wear 106 (1985) 5-33.

6. R. Rajasekaran, D. Nowell, Fretting fatigue in dovetail blade roots: Experiment and analysis, Tribology International 39 (2006) 1277-1285.

7. M. Ciavarella, G. Demelio, A review of analytical aspects of fretting fatigue, with extension to damage parameters, and application to dovetail joints, International Journal of Solids and Structures 38 (2001) 1791-1811.

8. J. Ding, G. Bandak, S.B. Leen, et al. Experimental characterization and numerical simulation of contact evolution effect on fretting crack nucleation for Ti-6Al-4V, Tribology International 42 (2009) 1651-1662.

9. A.L. Mohd Tobi, J. Ding, G. Bandak, S.B. Leen, P.H. Shipway, A study on the interaction between fretting wear and cyclic plasticity for Ti-6Al-4V, Wear 267 (2009) 270-282.

10. V.L. Popov, M. Heß, Method of dimensionality reduction in contact mechanics and friction. Springer-Verlag, Berlin Heidelberg (2015).

11. M. Heß, V.L. Popov, Method of Dimensionality Reduction in Contact Mechanics and Friction: A User's Handbook. II. Power-Law Graded Materials. Facta Universitatis, Series: Mechanical Engineering 14(3) (2016) 251-268.

12. M. Ciavarella, D.A. Hills, Brief Note: Some observations on the Oscillating Tangential Forces and Wear in General Plane Contacts, European Journal of Mechanics - A/Solids 18 (1999) 491-497.

13. J. Hintikka, A. Lehtovaara, C. Lönnqvist, Effect of start-up schemes and amplitude of tangential motion on friction behavior in fretting point contact, Tribology International 44(11) (2011) 1535-1543. 
14. A. Pannemaecker, H. Attia, G. Williams, A novel acceleration-controlled random vibration fretting test methodology: From classical sinusoidal to Gaussian random excitation, Wear 438-439 (2019) 203050.

15. F. Pompanon, J. Laporte, S. Fouvry, O. Alquier, Normal force and displacement amplitude influences on silver-plated electrical contacts subjected to fretting wear: A basic friction energy - contact compliance formulation, Wear 426-427 Part A (2019) 652-661.

16. I.I. Argatov, Y.S. Chai, An artificial neural network supported regression model for wear rate, Tribology International 138 (2019) 211-214.

17. A.V. Dimaki, A.I. Dmitriev, Y.S. Chai, V.L. Popov, Rapid simulation procedure for fretting wear on the basis of the method of dimensionality reduction, International Journal of Solids and Structures 51 (2014) 4215-4220.

18. Z. Xu, J. Peng, J. Liu, X. Liu, W. Zhang, M. Zhu, Study on fretting wear and tribo-chemical behavior of LZ50 axle steel in torsional fretting fatigue, Wear 426-427 Part A (2019) 704711

19. J. Peng, X. Jin, Z. Xu, J. Zhang, Z. Cai, Z.Luo, M.Zhu, Study on the damage evolution of torsional fretting fatigue in a 7075 aluminum alloy, Wear 402-403 (2018) 160-168.

20. S. Gao, Z. Cai, H. Quan, M. Zhu, H. Yu, Comparison between radial fretting and dualmotion fretting features of cortical bone, Tribology International 43(1-2) (2016) 440-446.

21. D.J. Lombardo, M.P. Siljander, C.K. Gehrke, D.D. Moore, E.A. Bakerand, Fretting and Corrosion Damage of Retrieved Dual-Mobility Total Hip Arthroplasty Systems, The Journal of Arthroplasty 34(6) (2019) 1273-1278.

22. Y. Zhou, M.X. Shen, Z.B. Cai, J.F. Peng, M.H. Zhu, Study on dual rotary fretting wear behavior of Ti6Al4V titanium alloy, Wear 376-377 Part A (2017) 670-679.

23. V.L. Popov, Analytic solution for the limiting shape of profiles due to fretting wear, Scientific Reports 4 (2014) 3749.

24. A.I. Dmitriev, L. Voll B., S.G. Psakhie, V.L. Popov, Universal limiting shape of worn profile under multiple-mode fretting conditions: theory and experimental evidence, Scientific Reports 6 (2016) 23231.

25. V.L. Popov, M. Heß, Method of Dimensionality Reduction in Contact Mechanics and Friction: A Users Handbook. I. Axially-Symmetric Contacts. Facta Universitatis, Series: Mechanical Engineering 12(1) (2014) 1-14.

26. L.D. Landau, E.M. Lifshitz, Course of Theoretical Physics, V. 9: Statistical Physics, Part. 2, Pergamon Press, Oxford, Second Edition, 1981 ( $\$ 60$ : Symmetry of electron states in a lattice in a magnetic field") 